\title{
Research on optimal allocation of energy storage capacity of microgrid considering various factors
}

\author{
Ning $\mathrm{Hu}^{1, \text { a }}$, Juncheng $\mathrm{Si}^{2}$, b , Yuanyuan Wang ${ }^{3, \mathrm{c}}$, Dehua Wang ${ }^{4,}$, , Hanghang Liu ${ }^{5, \mathrm{e}}$, Guanglei $\mathrm{Li}^{6, \mathrm{f}}$ \\ ${ }^{1}$ State Grid Shandong Power Supply Company \\ State Grid Dongying Power Supply Company Dongying, China \\ ${ }^{2}$ State Grid Shandong Power Supply Company \\ State Grid Dongying Power Supply Company Dongying, China \\ ${ }^{3}$ State Grid Shandong Power Supply Company \\ State Grid Dongying Power Supply Company Dongying, China \\ ${ }^{4}$ State Grid Shandong Power Supply Company \\ State Grid Shandong Electric Power Research Institute Jinan, China \\ ${ }^{5}$ State Grid Shandong Power Supply Company \\ State Grid Dongying Power Supply Company Dongying, China \\ ${ }^{6}$ State Grid Shandong Power Supply Company \\ State Grid Shandong Electric Power Research Institute Jinan, China
}

\begin{abstract}
For the grid connected by high proportion distributed power, energy storage system can effectively reduce the adverse impact of micro grid power fluctuation on the grid. Because of the high cost of energy storage system, it is necessary to optimize the capacity of energy storage system. Therefore, this paper proposes a capacity optimization method of energy storage system. Based on the comprehensive consideration of system objectives, construction costs, energy storage security and other factors, the simulation analysis model of the system is established, and the control strategy is optimized. In this paper, the algorithm is applied to the construction of microgrid demonstration project. The experimental results verify the effectiveness of the proposed method.
\end{abstract}

\section{INTRODUCTION}

Renewable energy power generation represented by wind power generation and photovoltaic power generation has the characteristics of intermittence and randomness. With the increasing penetration of renewable energy, it brings more and more challenges to the safe and reliable operation of power grid. Microgrid is composed of renewable energy power supply system, controllable power supply, energy storage system and load, which provides an effective technical way for high permeability renewable energy grid connection[1]. Due to the high cost of energy storage, it is necessary to consider various factors in combination with the requirements of power grid operation control. In the process of system construction, it is necessary to consider the economic investment restrictions, technical reliability restrictions and other factors. It is necessary to optimize the capacity allocation of energy storage system, and finally determine a reasonable construction scale.

In literature[2,3], the improved foraging algorithm such as cell foraging algorithm and genetic algorithm were used to enhance the global search ability and speed up the process of power capacity optimization. Literature [4] used Homer software to model the distributed power and load in the microgrid, and obtained the optimal power configuration scheme to meet the constraints. However, Homer's built-in algorithm does not consider the utilization of renewable energy, resulting in configuration winds and light. Literature [5] analyzed the probability statistics law of short-term forecast error of photovoltaic output and short-term forecast error of load, and used the interval estimation method to get the capacity allocation function of energy storage equipment.

In literature[6,7], using the scenario probability method, the expected cost of micro-grid investment operation is minimized by optimizing the energy storage output and diesel generator output within a certain period of time. However, the amount of calculation rapidly increases with the increase of the number of scenarios and the optimization time is too long. Literature $[7,8]$ proposed a multi-objective quantitative optimal configuration method of wind and solar power supply system based on probability model. The multiplicative optimization model was established with the maximum of

\footnotetext{
asddyhn19@sohu.combdysjc@163.com cdlwyy@sohu.com dsddlwdhl@126.com edylhh@126.com

flovexjtulgl@126.com
} 
power supply, the total investment of power supply system and the minimum voltage over-limit probability as the optimization objective. Monte Carlo method and the upper sub-point method were used to solve the capacityconstrained capacity allocation model [9,10]. The practical examples proved the effectiveness of the method.

As the country's emphasis on renewable energy development and implementation of the renewable energy Law, more and more wind farms in China access to the power system. For wind farms accessed to the big electric network, the scale of wind farms will not be not restricted when installed wind capacity accounted for the proportion of total installed capacity of less than $10 \%$. For the wind farms accesses to the electric distribution network, it is located in the area where have small load and the network structure is weakness that wind speed fluctuation, fault and other disturbance will cause great influence to the wind farm and the reliable operation of the local power grid. In order to be able to make ensure the safe and stable operation of the system,we should do calculation and analysis of stability of the region distribution network which accessed wind power.

Because of the intermittent and random characteristics of wind power, it is difficult to accurately predict the output. Energy storage can calm down wind power output fluctuations and improve power quality. Energy storage systems can reduce abandoned wind. From a technical point of view, energy storage is the powerful means to ensure the consumption of offshore wind power, but currently economics is the main factor restricting the matching of offshore wind power with energy storage.

\section{Calculation model}

\subsection{Charge percentage constraint}

Battery system remaining charge percentage constraint can be expressed as follows.

$$
\begin{gathered}
\operatorname{SOC}(t)=\frac{E_{\text {bat }}(t)}{N_{\text {bat }} E_{\text {bat,max }}} \\
\mathrm{SOC}_{\text {min }} \leqslant \operatorname{SOC}(t) \leqslant \mathrm{SOC}_{\text {max }}
\end{gathered}
$$

In the above formula, $\operatorname{SOC}(t)$ is the percentage of remaining battery capacity of the battery system, $E_{\mathrm{bat}}(t)$ is the standard single-battery rated capacity $(\mathrm{kW} \cdot \mathrm{h})$, $\mathrm{SOC}_{\max }, \mathrm{SOC}_{\min }$ are the upper and lower limits of the percentage of remaining battery capacity.

\subsection{Battery float life}

In order to simplify the problem, we consider that the design life of the storage battery system is determined by two factors: the cumulative charge and discharge capacity. The battery float life can be expressed as follows.

$$
\begin{array}{r}
L_{\text {bat }}=\min \left(\frac{N_{\text {bat }} Q_{\text {bat }}}{Q_{\text {thrpt }}}, L_{\text {bat }, \mathrm{f}}\right) \\
Q_{\text {thrpt }}=\sum_{t=1}^{T} P_{\text {bat_c }}(t) \eta_{\mathrm{c}}+\sum_{t=1}^{T} \frac{P_{\text {bat_d }}(t)}{\eta_{\mathrm{d}}}
\end{array}
$$

In the above formula, $L_{\text {bat,f }}$ is the design life of the battery system (years), $Q_{\text {thrpt }}$ is the cumulative charge and discharge capacity of the single battery $(\mathrm{kW} \cdot \mathrm{h})$, $L_{\text {bat }}$ is the battery system float life (years), $\mathrm{T}$ is the total number of one-year hours, that is $8760 \mathrm{~h}$.

The model assumes that there is an upper limit $\mathrm{Q}$ for the cumulative charge and discharge capacity of a single battery. That is, the more charge and discharge capacity, the less remaining charge available, and the closer the battery is to its upper limit of life. It can define a battery wear indicators, Used to indicate the proportion of energy storage batteries consumed over a period of time in the total battery capacity, that is expressed as follows.

$$
\kappa=\max \left\{\frac{Q_{\text {thrpt }}}{N_{\text {bat }} Q_{\text {bat }}}, \frac{1}{L_{\text {bat }, \mathrm{f}}}\right\}
$$

In this paper, the standard rated capacity of $6 \mathrm{~kW} \cdot \mathrm{h}$, rated power of $1.2 \mathrm{~kW}$, the cumulative charge and discharge capacity ceiling $Q_{\mathrm{bat}}$ is $10196 \mathrm{~kW} \cdot \mathrm{h}$, charge and discharge efficiency of $86 \%$, The maximum discharge depth of $70 \%$, floating life of 20 years, the battery leakage rate of $0.01 \%$.

\section{ECONOMIC OPTIMIZATION MODEL}

\subsection{The objective function}

Unlike energy storage batteries, fans, photovoltaic panels and diesel generators are replaced at the end of their lives, and the engineering cycle is not necessarily an integral multiple of the design life. Therefore, annual replacement costs need to be taken into account when equipment residuals, fan replacement costs The formula is shown as follows..

$$
C_{\mathrm{RW}}=N_{\mathrm{WT}} C_{\mathrm{rw}} f_{\mathrm{wr}} \mathrm{SFF}\left(r, L_{\mathrm{W}}\right)-S_{\mathrm{w}} \operatorname{SFF}(r, L)
$$

Among them, the main parameters are calculated as follows. 


$$
\begin{aligned}
f_{\mathrm{wr}}= & \begin{array}{ll}
\operatorname{CRF}(r, L) / \operatorname{CRF}\left(r, L_{\mathrm{wrep}}\right) & L_{\mathrm{wrep}}>0 \\
0 & L_{\mathrm{wrep}}=0
\end{array} \\
& L_{\mathrm{wrep}}=L_{\mathrm{W}} \mathrm{INT}\left(\frac{L}{L_{\mathrm{W}}}\right) \\
& S_{\mathrm{w}}=N_{\mathrm{wT}} C_{\mathrm{rw}} \frac{L_{\mathrm{wrem}}}{L_{\mathrm{W}}} \quad L_{\mathrm{wrem}}=L_{\mathrm{W}}-\left(L-L_{\mathrm{wrep}}\right)
\end{aligned}
$$

Where, $\operatorname{CRF}\left(r, L_{\text {wrep }}\right)$ is the fan replacement cost (Yuan/Taiwan). $f_{\mathrm{wr}}$ is the design life of the fan (year). Integer INT $(\mathrm{x})$ is the largest integer less than or equal to $\mathrm{x}, \mathrm{L}$ is the fan design of the project cycle Lifetime and the number of replacements, $S_{w}$ is the remnant of the remaining equipment of the fan at the end of the project cycle, $\mathrm{L}$ is the remaining life of the fan at the end of the project cycle. Similarly, the annual replacement costs of photovoltaic and diesel generators can be calculated as $C_{\mathrm{rw}}$.

When the operation load of the power grid is in the trough stage and the wind power output is large, the superconducting energy storage device can reserve excess electrical energy. In addition, when the power grid is operating during peak periods or when wind power output is low, the energy storage device sends stored electrical energy to the power grid. Energy storage devices can be used not only for peaks and valleys of the power system, but also for their fast response speed, which can play a certain role in eliminating or reducing low frequency oscillations in the power grid, thereby improving the frequency characteristics and voltage stability of the power grid. In addition, the energy storage device can also adjust the power factor, thereby improving the stability of the power system.

\subsection{Constraints}

(1) Proportion of installed capacity of renewable energy In order to make full use of the island's abundant renewable energy resources and reduce the environmental pollution caused by traditional fuels, the proportion of renewable energy installed capacity $f$ shall not be less than its lower limit $\mathrm{f}$

$$
f_{\mathrm{thr}}=\frac{N_{\mathrm{WT}} P_{\mathrm{WT}}+N_{\mathrm{PV}} P_{\mathrm{STC}}}{L_{\mathrm{max}}} \geqslant f_{\mathrm{thr}, \text { min }}
$$

In the formula, $f_{\text {thr }}$ is the proportion of renewable energy installed capacity, $f_{\text {thr,min }}$ is the proportion of the minimum installed capacity of renewable energy, and $L_{\max }$ is the annual peak load $(\mathrm{kW})$.

(2) power supply reliability rate constraints

In some extreme cases, the sum of distributed power outputs can not meet the load requirements, but it is required to cut the load partially. However, it is required that the total annual load shedding can not exceed the LPSP Upper limit value $f_{\text {LPSP }}$.The smaller the upper limit, the higher the system reliability requirements.

$$
\sum_{t=1}^{T} P_{\text {loss }}(t) \leqslant f_{\text {LPSP }} \sum_{t=1}^{T} \operatorname{load}(t)
$$

\section{EXAMPLE ANALYSIS}

The data of wind speed, light intensity, temperature and load of the region in 2011 are shown in Figure 1. Consider five kinds of distributed power combination scheme of scenery storage, scenery firewood, light storage firewood, wind firewood and scenery firewood, apply Matlab and call the MILP solution function in CPLEX to solve the optimal capacity configuration results of the five solutions separately. Table 1 shows several types of distributed power cost data.

TABLE I. OPTIMAL CONFIGURATION PARAMETERS OF ENERGY STORAGE SYSTEM

\begin{tabular}{|c|c|c|c|c|}
\hline $\begin{array}{c}\text { Energy } \\
\text { storage } \\
\text { type }\end{array}$ & $\begin{array}{c}\text { Working } \\
\text { frequency } \\
\text { band/ } \\
\mathbf{m H z}\end{array}$ & $\begin{array}{c}\text { Rated } \\
\mathbf{p o w e r} / \\
\mathbf{k w}\end{array}$ & $\begin{array}{c}\text { Rated } \\
\text { capacity/ } \\
\mathbf{k W h}\end{array}$ & $\begin{array}{c}\text { State of } \\
\text { charge/ } \\
\mathbf{\%}\end{array}$ \\
\hline $\begin{array}{c}\text { lithium } \\
\text { battery }\end{array}$ & $0.03-1.68$ & 316.45 & 108.25 & $56.26 \%$ \\
\hline $\begin{array}{c}\text { Super } \\
\text { capacitor } \\
\text { energy } \\
\text { storage }\end{array}$ & $1.68-17.76$ & 946.28 & 425.16 & $84.36 \%$ \\
\hline
\end{tabular}

The installed capacity of renewable energy exceeds the peak value of load in all of the five configurations. This is because the fluctuation of scenery output is larger and the probability of full power generation is smaller. If the capacity of renewable energy is configured according to the load peak, it is often difficult to meet the load Electricity demand.

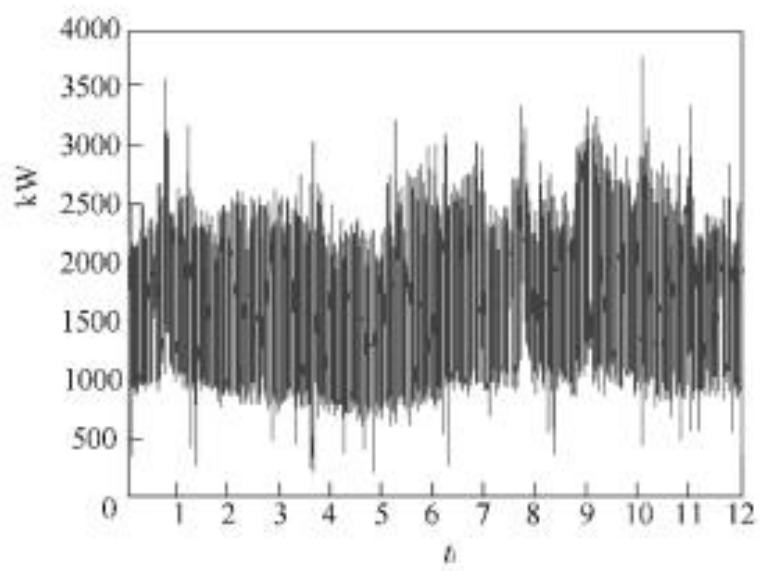

Figure 1. Microgrid annual load curve

It is necessary to build the microgrid to improve the utilization of electricity. It can transform renewable resources into electrical energy and store it in batteries. There will be peak and trough periods in the process of user power consumption. Microgrid can be used to more reasonably and scientifically adjust the order of power supply. From the changing trend of microgrid operation, it can be found that the system's economic benefits can be brought into full play during the actual operation of the microgrid. In the state where the distribution network is 
connected to the microgrid, the best economic benefits of microgrid operation are obtained.

\section{CONCLUSION}

Because of the high cost of energy storage system, it is necessary to optimize the capacity of energy storage system. Therefore, different kinds of distributed power sources Programs are becoming more economical and environmentally friendly. In order to prolong the life time of energy storage battery, it is not economical to maintain its SOC with the high level during micro-grid operation. This will help prolong the service life of energy storage battery, it will reduce its usable capacity and increase the storage capacity.

\section{ACKNOWLEDGMENT}

This work was supported by Science and technology project of State Grid Shandong Power Supply Company.

\section{REFERENCES}

1. Lu Zongxiang, Wang Caixia et al, " Overview on microgrid researc," Automation of Electric Power Systems, vol. 31, pp. 104-111, October 2007.

2. Zhang Jianhua, Yu Lei et al, " Optimization of capacity of microgrid with wind/light/diesel/impoundment and seawater desalination load," Transactions of China Electrotechnical Society, vol. 29, pp. 102-112, June 2014.

3. Yu Peng, Liu Xinghua et al, " Study on operation control of island microgrid with high renewable energy penetration," Power System Technology, vol. 42, pp. 779-788, August 2018

4. Ma Xiyuan, Wu Yaowen et al, “ Optimization of power supply for wind/light/storage hybrid microgrid with improved bacteria foraging algorithm," Proceedings of the CSEE, vol. 31, pp. 17-25, June 2011

5. Wan Chengshan, Wu Zhen et al, " Research on key technologies of microgrid," Transactions of China Electrotechnical Society, vol. 29, pp. 1-9, August 2014

6. Wang Zhaoyu, Ai Qian et al, “ Multi-objective allocation of microgrid in smart distribution network," Power System Technology, vol. 36, pp. 200-203, June 2012.

7. Ye Chengjin, Huang Minxiang et al, " Optimization of wind and solar power system based on discrete probability model," Automation of Electric Power Systems, vol. 37, pp. 48-54, March 2013.

8. Ma Rui, Jin Yan et al, “ An Optimal Dual-layer Configuration of Active Distribution Network Based on Chance-constrained Programming," Transactions of China Electrotechnical Society, vol. 31, pp. 145-154, June 2016.

9. Liu Ziqiu, Huang Mingxiang et al, “ Optimal energy allocation of microgrid based on reliability and economy by considering characteristics of micro source," Power System Technology, vol. 38, pp. 1352-1357, June 2014.

10. Dai Juchuan, Liu Deshun et al, “ Research on power coefficient of wind turbines based on SCADA data," Renewable Energy, vol. 86, pp. 206-215, October 2016. 